\title{
Deuterium Isobaric Amine Reactive Tag
}

National Cancer Institute

\section{Source}

National Cancer Institute. Deuterium Isobaric Amine Reactive Tag. NCI Thesaurus. Code C161873.

A reagent used for isobaric mass labeling that is amine reactive and tagged with the heavy hydrogen isotope deuterium. Deuterium isobaric amine reactive tags have been used in phosphoproteomic studies. 